VPI-IHEP-92-4

hepth@xxx/9203014

\title{
BRST Cohomology and Invariants of 4D Gravity in Ashtekar Variables
}

\author{
Lay Nam Changl \& Chopin Sod2 \\ Institute for High Energy Physics \\ Virginia Polytechnic Institute and State University \\ Blacksburg, Virginia 24061-0435
}

\begin{abstract}
We discuss the BRST cohomologies of the invariants associated with the description of classical and quantum gravity in four dimensions, using the Ashtekar variables. These invariants are constructed from several BRST cohomology sequences. They provide a systematic and clear characterization of non-local observables in general relativity with unbroken diffeomorphism invariance, and could yield further differential invariants for fourmanifolds. The description includes fluctuations of the vierbein fields, but there exits a non-trivial phase which can be expressed in terms of Witten's topological quantum field theory. In this phase, the descent sequences are degenerate, and the corresponding classical solutions can be identified with the conformally self-dual sector of Einstein manifolds. The full theory includes fluctuations which bring the system out of this sector while preserving diffeomorphism invariance.
\end{abstract}

$3 / 92$

\footnotetext{
1 e-mail:laynam@vtvm2.bitnet

2 e-mail:soocp@vtcc1.bitnet
} 
A great deal has been learned recently about 2-manifolds, and it is now possible to give a complete description in terms of the gravitational fields in two dimensions (2D) [1]. Similar results have also been obtained in three dimensions [2]. It is however not entirely clear how such results can be extended to 4-manifolds. There are several reasons for suspecting that this generalization will not be straightforward. Firstly, unlike in two and three dimensions, in four dimensions pure gravity can exist in a phase wherein there are two propagating degrees of freedom. Secondly, the work of Donaldson and others[3] shows that 4-manifolds can have far richer differential structures than in other dimensions, so their complete characterization can prove to be a daunting task indeed.

Witten has suggested [4] [5at $4 \mathrm{D}$ gravity possesses a phase describable by a topological quantum field theory(TQFT)[6], in that the observables consist entirely of global invariants. Some of these invariants have been identified. In particular, it can be shown that by considering the moduli space of (anti-) instantons, the Donaldson maps [7] can be identified as BRST invariants of the corresponding TQFT [4].

What happens when we have 4-manifolds which can support 4D gravity with propagating degrees of freedom? To extend Witten's analysis to such instances, it will be necessary to describe these degrees of freedom using variables that are related naturally to those employed in TQFT. In particular, we must require that they are suitable for implementing both diffeomorphism and gauge invariance.

In this article, we show that in many respects the variables introduced by Ashtekar satisfy these requirements [8]. In place of the metric of general relativity, the classical Ashtekar variables corresponding to specific Einstein manifolds consist of densitized triad fields, and their conjugate momenta, which turn out to be simply $S O(3)$ gauge potentials satisfying (anti-) self-duality conditions. We shall analyze the classical and BRST symmetry of 4D gravity in terms of these variables, determine their cohomology descent equations, and identify the invariants which can be constructed out of them. These quantities can then be used to describe the diffeomorphism and gauge invariant observables of the theory, and to furnish characterizations of the differential structures of such 4-manifolds.

We shall work with Riemannian manifolds and start with the action proposed by Samuel[9]:

$$
\mathcal{A}=\frac{1}{16 \pi G} \int_{M}\left(2 F_{a} \wedge \Sigma_{a}+\frac{\lambda}{3} \Sigma_{a} \wedge \Sigma_{a}\right)
$$

where the anti self-dual two-form

$$
\Sigma_{a}=\frac{1}{2} \epsilon_{a}^{b c}\left\{e_{b} \wedge e_{c}-e_{0} \wedge e_{a}\right\}
$$


and $e_{A}, A=0, \ldots, 3$ denote the vierbein one-forms in four dimensions. The Latin indices run from 1 to 3 and label internal $S O(3)$ indices. $F_{a}$ is the curvature two-form of the $S O(3)$ Ashtekar connection $A_{a}$, while $\lambda$ is the cosmological constant. In the Ashtekar formalism, the metric is considered to be a derived quantity, expressed in terms of $\Sigma$ through

$$
\tilde{g}_{\mu \nu}=\frac{1}{12} \epsilon_{a b c} \tilde{\epsilon}^{\alpha \beta \gamma \delta} \Sigma_{\alpha \beta}^{a} \Sigma_{\gamma_{\mu}}^{b} \Sigma_{\nu \delta}^{c}
$$

In applying the canonical formalism to Eqn. (1), it is convenient to work in the spatial gauge in which the vierbein can be written as

$$
e_{A \mu}=\left[\begin{array}{cc}
N & 0 \\
N^{j} e_{a j} & e_{a i}
\end{array}\right]
$$

where the Greek index $\mu$ runs from 0 to 3 . The form assumed in (4) is compatible with the ADM[10] decomposition of the metric

$$
\begin{aligned}
d s^{2} & =e_{A \mu} e_{A \nu} d x^{\mu} d x^{\nu} \\
& =N^{2}\left(d x^{0}\right)^{2}+g_{i j}\left(d x^{i}+N^{i} d x^{0}\right)\left(d x^{j}+N^{j} d x^{0}\right)
\end{aligned}
$$

with the spatial metric $g_{i j}=e^{a}{ }_{i} e_{a j}$. Thus we see that the choice (丑) in no way compromises the values of the lapse and shift functions $N$ and $N^{i}$ which have geometrical interpretations in hypersurface deformations. With this decomposition, it is straightforward to re-write Eqn. (11) as

$$
\begin{aligned}
\mathcal{A}=\frac{1}{16 \pi G} & \int d^{4} x\left\{2 \tilde{\sigma}^{i a} \dot{A}_{i a}+2 A_{0 a} D_{i} \tilde{\sigma}^{i a}+2 N^{j} \tilde{\sigma}^{i a} F_{i j a}\right\} \\
& -\frac{1}{16 \pi G} \int d^{4} x\left\{N\left(\epsilon_{a b c} \tilde{\sigma}^{i a} \tilde{\sigma}^{j b} F_{i j}^{c}+\frac{\lambda}{3} \epsilon_{a b c} \epsilon_{\sim j k} \tilde{\sigma}^{i a} \tilde{\sigma}^{j b} \tilde{\sigma}^{k c}\right)\right\} \\
& + \text { boundary terms }
\end{aligned}
$$

with $\tilde{\sigma}$ and $\underset{\sim}{\sim}$ defined as follows

$$
\begin{aligned}
\tilde{\sigma}^{i a} & \equiv \frac{1}{2} \tilde{\epsilon}^{i j k} \epsilon^{a b c} e_{j b} e_{k c} \\
\stackrel{N}{\sim} & \equiv \operatorname{det}\left(e_{a i}\right)^{-1} N
\end{aligned}
$$

The tildes above and below the variables indicate that they are tensor densities of weight 1 and -1 respectively. Thus $2 \tilde{\sigma}^{i a}$ is readily identified as the conjugate variable to $A_{i a}$. (We shall suppress the factor $16 \pi G$ for convenience). The variables $A_{0 a}, N^{i}$ and $\underset{\sim}{N}$ are clearly 
Lagrange multipliers for the Ashtekar constraints. These constraints can be identified as Gauss' law generating $S O(3)$ gauge invariance

$$
G^{a} \equiv 2 D_{i} \tilde{\sigma}^{i a} \approx 0
$$

and the "supermomentum" and "superhamiltonian" constraints

$$
\begin{aligned}
H_{i} & \equiv 2 \tilde{\sigma}^{j a} F_{i j a} \approx 0 \\
H & \equiv \epsilon_{a b c} \tilde{\sigma}^{i a} \tilde{\sigma}^{j b}\left(F_{i j}^{c}+\frac{\lambda}{3} \epsilon_{i j k} \tilde{\sigma}^{k c}\right) \approx 0
\end{aligned}
$$

Ashtekar showed that these constraints, despite their remarkable simplicity, are equivalent in content to the constraints and constraint algebra of $4 D$ general relativity. The equations of motion that are obtained can be written succinctly as [1]

$$
\begin{aligned}
D \Sigma_{a} & =0 \\
F_{a} & =S_{a b} \Sigma^{b}
\end{aligned}
$$

with

$$
S_{a b}=S_{b a} \quad \text { and } \quad \operatorname{Tr} S=-\lambda
$$

The last two conditions solve the "supermomentum" and "superhamiltonian" constraints, and can be taken to be the general solution for non-degenerate metrics. For metrics of Euclidean signature, $S$ is a real symmetric matrix with three eigenvectors. The classical solutions can then be classified according to the number of distinct eigenvalues of $S$, and are called Types I, D, or O, depending on there being three, two, or one, distinct eigenvalues respectively. The set of equations (10 ) can be shown to be equivalent to the equations for Einstein manifolds in four dimensions i.e. $R_{\mu \nu}=\lambda g_{\mu \nu}$. One can, in principle, eliminate $S$ from the theory by using the relation implied in $(10 b)$ and obtain

$$
S_{a b}=-\frac{1}{2} *\left(F_{a} \wedge \Sigma_{b}\right)
$$

with

$$
\epsilon_{a}^{b c} F_{b} \wedge \Sigma_{c}=0
$$

3 We show elsewhere 111] that all classical Einstein manifolds can be described as anti-instantons in the Ashtekar variables. Indeed, we can establish a relationship between the reduced phase space of these variables and the moduli space of reducible and irreducible anti-instantons, and thence construct new topologically non-trivial solutions. 
and

$$
F_{a} \wedge \Sigma_{a}=-2 \lambda *(1)
$$

where $*$ is the hodge dual operator. $S$ plays an important role in characterizing the possible phases of the theory. However, it is prudent not to eliminate $\Sigma$ in favor of $F$ by inverting $S$, because there are various interesting cases for which $S$ is non-invertible, and yet the vierbein and $\Sigma$ remain regular. Explicit examples, especially those involving the $F=0$ sector, as well as cases with abelian anti-instantons, are described in [11]. In what follows, we shall construct invariants that are dependent on $F$ and on $\Sigma$, thereby demonstrating that they are both essential in the description of $4 \mathrm{D}$ gravity.

We begin by analyzing the symmetries of the action and the associated BRST invariance. It is easy to see that the action is invariant under $S O(3)$ gauge transformations as well as four dimensional diffeomorphisms. Working with $\mathcal{A}$, which is explicitly gaugeinvariant, we can consider a diffeomorphism $\phi$, of $M$ into itself, generated by the vector field $\beta$. On the one-form variables $A_{a}$ and $e_{A}$, the induced variations are Lie derivatives

$$
\delta A_{a}=\mathcal{L}_{\beta} A_{a}=\left(i_{\beta} d+d i_{\beta}\right) A_{a}
$$

and

$$
\delta e_{A}=\mathcal{L}_{\beta} e_{A}
$$

with $i_{\beta}$ denoting interior multiplication or contraction with the vector field $\beta$, which in local coordinates can be written as $\beta^{\mu} \partial_{\mu}$. If we denote the Lagrangian four-form as $L$, then

$$
\begin{gathered}
\int_{M}\left(L-\phi^{*} L\right)=0 \\
\Longleftrightarrow \int_{M} \mathcal{L}_{\beta} L=\int_{\partial M} i_{\beta} L=0
\end{gathered}
$$

The action is thus invariant if $M$ is closed, while for open $M$, invariance can be maintained provided $\beta$ vanishes at the boundary. Since $S O(3)$ gauge invariance is a symmetry of the theory, one can also consider diffeomorphisms for which the exterior derivative operator in the Lie derivative is replaced by the covariant derivative to make it compatible with the canonical analysis. 
In the BRST formalism, the classical gauge and diffeomorphism symmetries are mimicked by transformations with the parameters replaced by ghosts $\eta_{a}^{\prime}$ and $\xi$. Thus the BRST transformations of the variables are (henceforth $\delta$ shall mean $\delta_{B R S T}$ )

$$
\begin{aligned}
\delta A_{a} & =-D \eta_{a}^{\prime}+\mathcal{L}_{\xi} A_{a} \\
\delta e_{a} & =-\epsilon_{a}{ }^{b c} \eta_{b}^{\prime} e_{c}+\mathcal{L}_{\xi} e_{a} \\
\delta e_{0} & =\mathcal{L}_{\xi} e_{0}
\end{aligned}
$$

For a general differential form $\chi$,

$$
\mathcal{L}_{\xi} \chi=\left(i_{\xi} d-d i_{\xi}\right) \chi
$$

There is a sign difference in the second term because, unlike a normal vector field, $\xi$ carries a ghost number of 1 . The above BRST transformation of the vierbein implies that the antiself-dual two form $\Sigma_{a}$ transforms according to

$$
\delta \Sigma_{a}=-\epsilon_{a}^{b c} \eta_{b}^{\prime} \Sigma_{c}+\mathcal{L}_{\xi} \Sigma_{a}
$$

The standard procedure of splitting the ghost $\eta^{\prime}$ into $\eta^{\prime}=\eta-i_{\xi} A$ allows us to write the BRST transformations in a gauge-covariant manner

$$
\begin{aligned}
& \delta A_{a}=-D \eta_{a}+i_{\xi} F_{a} \\
& \delta \Sigma_{a}=-\epsilon_{a}^{b c} \eta_{b} \Sigma_{c}+i_{\xi} D \Sigma_{a}-D i_{\xi} \Sigma_{a}
\end{aligned}
$$

The BRST transformations for the ghosts are

$$
\delta \eta_{a}=-\frac{1}{2} \epsilon_{a}^{b c} \eta_{b} \eta_{c}+\frac{1}{2} i_{\xi} i_{\xi} F_{a}
$$

and

$$
\delta \xi=\frac{1}{2} \mathcal{L}_{\xi} \xi \quad \text { i.e. } \quad \delta \xi^{\mu}=\xi^{\nu} \partial_{\nu} \xi^{\mu}
$$

It can be verified that the BRST transformations above are nilpotent i.e. $\delta^{2}=0$. The variables $(A, \eta, \Sigma, \xi)$, which are $(1,0,2)$ forms and a vector-field respectively, are assigned ghost numbers $(0,1,0,1)$ and carry a grading equal to the form degree plus the ghost number. By this we mean that if $\chi_{1,2}$ are $p_{1,2}$-forms with ghost numbers $g_{1,2}$, then

$$
\chi_{1} \wedge \chi_{2}=(-1)^{p} \chi_{2} \wedge \chi_{1} ; \quad p=\left(p_{1}+g_{1}\right)\left(p_{2}+g_{2}\right)
$$


The effective quantum action will consist of the classical action and a piece from a gauge-fixing Lagrangian of the form $L_{g . f .}=\delta \chi$ which will involve anti-ghosts and auxiliary fields. Since the BRST transformation is nilpotent, the effective action will be BRSTinvariant provided the boundary term vanishes.

The transformation rules for $A$ and $\eta$ may also be obtained by considering the multiplet $(A, \eta)$ as a connection $\widetilde{A}$ of the universal bundle over $M \times \mathcal{C} / \mathcal{G}$. Here $\mathcal{C}$ is the space of connections $A$, while $\mathcal{G}$ is the group of gauge transformations. $\widetilde{A}$ carries a grading of 1 , and can be decomposed into its $(1,0)$ and $(0,1)$ components as

$$
\widetilde{A}_{a}=A_{a}+\eta_{a}
$$

Its curvature is given by

$$
\widetilde{F}_{a}=(d+\delta) \tilde{A}_{a}+\frac{1}{2} \epsilon_{a}{ }^{b c} \widetilde{A}_{b} \wedge \widetilde{A}_{c}
$$

The transformation rules for $A$ and $\eta$ are equivalent to the statement

$$
\widetilde{F}_{a}=\exp \left(i_{\xi}\right) F_{a}=\left(I+i_{\xi}+\frac{1}{2 !} i_{\xi} i_{\xi}\right) F_{a}
$$

which reduces to the "soul-flatness" condition, $\widetilde{F}=F$, in ordinary gauge theories when diffeomorphism invariance generated by $\xi$ is absent. Here the concept of "horizontality" in curved space is the statement that $\widetilde{F}$ can be expanded in terms of $F$ and its contractions with the ghost $\xi$. The BRST transformation of $F$ as a consequence of (20a) is

$$
\delta F_{a}=-\epsilon_{a}^{b c} \eta_{b} F_{c}-\left(D i_{\xi} F\right)_{a}
$$

It can be verified that the curvature $\widetilde{F}$ satisfies the Bianchi identity

$$
\widetilde{D} \widetilde{F}_{a}=(d+\delta) \widetilde{F}_{a}+\epsilon_{a}{ }^{b c} \widetilde{A}_{b} \wedge \widetilde{F}_{c}=0
$$

As a consequence $\operatorname{Tr}\left(\widetilde{F}^{n}\right)$ obeys

$$
\widetilde{D}\left(\operatorname{Tr}\left(\widetilde{F}^{n}\right)\right)=0
$$

Since the gauge group is $S O(3)$, it suffices to consider $n=2$. One can expand $\widetilde{F}_{a} \wedge \widetilde{F}_{a}$ in terms of the ghost number i.e. writing

$$
\widetilde{F}_{a} \wedge \widetilde{F}_{a}=\sum_{g=0}^{4} W_{p=4-g}^{g}
$$


The resulting BRST cohomology descent equations from (29) i.e.

$$
(d+\delta)\left(\widetilde{F}_{a} \wedge \widetilde{F}_{a}\right)=0
$$

are

$$
\begin{aligned}
d W_{4}^{0} & =0 \\
\delta W_{4-g}^{g} & =-d W_{3-g}^{g+1} \quad \text { for } g=1,2,3 \\
\delta W_{0}^{4} & =0
\end{aligned}
$$

with

$$
\begin{array}{ll}
W_{4}^{0}=F_{a} \wedge F_{a} & W_{3}^{1}=i_{\xi}\left(F_{a} \wedge F_{a}\right) \\
W_{2}^{2}=\frac{1}{2 !} i_{\xi} i_{\xi}\left(F_{a} \wedge F_{a}\right) & W_{1}^{3}=\frac{1}{3 !} i_{\xi} i_{\xi} i_{\xi}\left(F_{a} \wedge F_{a}\right) \\
W_{0}^{4}=\frac{1}{4 !} i_{\xi} i_{\xi} i_{\xi} i_{\xi}\left(F_{a} \wedge F_{a}\right) &
\end{array}
$$

Consequently, there is an off-shell descent sequence involving the curvature of the Ashtekar connection. In view of the fact that on-shell, the covariant curl of $\Sigma$ is zero and $\Sigma$ transforms in the same way as $F$, one expects that a BRST cohomology sequence analogous to the $\mathrm{W}$ descent exists for $\Sigma$ as well. This is indeed true if one also makes use of the other equations of motion (10b) and (10c). This suggests that even off-shell, a descent involving $\Sigma$ could be realized. However for off-shell computations we should keep all terms involving $D \Sigma$. We thus find that the BRST transformations of $\Sigma$ and $\xi$, Eqns. (20) and (21) imply

$$
\begin{aligned}
\delta\left(i_{\xi} \Sigma_{a}\right) & =-\epsilon_{a}{ }^{b c} \eta_{b} i_{\xi} \Sigma_{c}+\frac{1}{2 !} i_{\xi} i_{\xi} D \Sigma_{a}-\frac{1}{2 !}\left[D\left(i_{\xi} i_{\xi} \Sigma\right)\right]_{a} \\
\delta\left(\frac{1}{2 !} i_{\xi} i_{\xi} \Sigma_{a}\right) & =-\epsilon_{a}{ }^{b c} \eta_{b} \frac{1}{2 !} i_{\xi} i_{\xi} \Sigma_{c}+\frac{1}{3 !} i_{\xi} i_{\xi} i_{\xi} D \Sigma_{a}
\end{aligned}
$$

So instead of the Bianchi identity for $\widetilde{F}$, we have

$$
\begin{aligned}
\widetilde{D} \widetilde{\Sigma}_{a}=(d+ & \delta)\left(\Sigma_{a}+i_{\xi} \Sigma_{a}+\frac{1}{2 !} i_{\xi} i_{\xi} \Sigma_{a}\right) \\
& +\epsilon_{a}^{b c}\left(A_{b}+\eta_{b}\right) \wedge\left(\Sigma_{c}+i_{\xi} \Sigma_{c}+\frac{1}{2 !} i_{\xi} i_{\xi} \Sigma_{c}\right) \\
= & D \Sigma_{a}+i_{\xi} D \Sigma_{a}+\frac{1}{2 !} i_{\xi} i_{\xi} D \Sigma_{a}+\frac{1}{3 !} i_{\xi} i_{\xi} i_{\xi} D \Sigma_{a}
\end{aligned}
$$

i.e.

$$
\widetilde{D} \widetilde{\Sigma}_{a}=\exp \left(i_{\xi}\right) D \Sigma_{a}
$$


Thus we see that the consistency condition is the requirement that $\widetilde{D} \widetilde{\Sigma}$ can be expanded in terms of $D \Sigma$ and its contractions with the ghost $\xi$. For $\widetilde{D} \widetilde{F}$, this expansion is trivial because of the Bianchi identity for $F$. By considering $\widetilde{D}\left(\widetilde{\Sigma}_{a} \wedge \widetilde{\Sigma}_{a}\right)$ we have

$$
(d+\delta)\left(\widetilde{\Sigma}_{a} \wedge \widetilde{\Sigma}_{a}\right)=2 \widetilde{D} \widetilde{\Sigma}_{a} \wedge \widetilde{\Sigma}_{a}
$$

and expanding $\widetilde{\Sigma}_{a} \wedge \widetilde{\Sigma}_{a}$ in terms of ghost number,

$$
\widetilde{\Sigma}_{a} \wedge \widetilde{\Sigma}_{a}=\sum_{g=0}^{4} V_{4-g}^{g}
$$

and using (208), (32) and (33), Eqn. (34) yields the identities

$$
\begin{aligned}
d\left(\Sigma_{a} \wedge \Sigma_{a}\right) & =2 D \Sigma_{a} \wedge \Sigma_{a} \\
\delta\left(\Sigma_{a} \wedge \Sigma_{a}\right) & =-d\left[i_{\xi}\left(\Sigma_{a} \wedge \Sigma_{a}\right)\right]+i_{\xi} d\left(\Sigma_{a} \wedge \Sigma_{a}\right) \\
\delta\left[i_{\xi}\left(\Sigma_{a} \wedge \Sigma_{a}\right)\right] & =-d\left[\frac{1}{2 !} i_{\xi} i_{\xi}\left(\Sigma_{a} \wedge \Sigma_{a}\right)\right]+\frac{1}{2 !} i_{\xi} i_{\xi} d\left(\Sigma_{a} \wedge \Sigma_{a}\right) \\
\delta\left[\frac{1}{2 !} i_{\xi} i_{\xi}\left(\Sigma_{a} \wedge \Sigma_{a}\right)\right] & =-d\left[\frac{1}{3 !} i_{\xi} i_{\xi} i_{\xi}\left(\Sigma_{a} \wedge \Sigma_{a}\right)\right]+\frac{1}{3 !} i_{\xi} i_{\xi} i_{\xi} d\left(\Sigma_{a} \wedge \Sigma_{a}\right) \\
\delta\left[\frac{1}{3 !} i_{\xi} i_{\xi} i_{\xi}\left(\Sigma_{a} \wedge \Sigma_{a}\right)\right] & =-d\left[\frac{1}{4 !} i_{\xi} i_{\xi} i_{\xi} i_{\xi}\left(\Sigma_{a} \wedge \Sigma_{a}\right)\right]+\frac{1}{4 !} i_{\xi} i_{\xi} i_{\xi} i_{\xi} d\left(\Sigma_{a} \wedge \Sigma_{a}\right) \\
\delta\left[\frac{1}{4 !} i_{\xi} i_{\xi} i_{\xi} i_{\xi}\left(\Sigma_{a} \wedge \Sigma_{a}\right)\right] & =-\frac{1}{5 !} i_{\xi} i_{\xi} i_{\xi} i_{\xi} i_{\xi} d\left(\Sigma_{a} \wedge \Sigma_{a}\right)
\end{aligned}
$$

It is remarkable that although $\Sigma$, unlike $F$, has a non-zero covariant curl off-shell, the nonexact terms on the R.H.S. of (35b) - (35f) actually vanish because they are all contractions with $\xi$ of $d\left(\Sigma_{a} \wedge \Sigma_{a}\right)$, which is zero in four dimensions. Hence, we do have another BRST cohomology sequence with descent equations

$$
\begin{aligned}
& d V_{4}^{0}=0 \\
& \delta V_{4-g}^{g}=-d V_{3-g}^{g+1} \quad \text { for } \quad g=1,2,3, \\
& \delta V_{0}^{4}=0
\end{aligned}
$$

where

$$
\begin{array}{rlrl}
V_{4}^{0} & =\Sigma_{a} \wedge \Sigma_{a} & V_{3}^{1}=i_{\xi}\left(\Sigma_{a} \wedge \Sigma_{a}\right) \\
V_{2}^{2}=\frac{1}{2 !} i_{\xi} i_{\xi}\left(\Sigma_{a} \wedge \Sigma_{a}\right) & V_{1}^{3}=\frac{1}{3 !} i_{\xi} i_{\xi} i_{\xi}\left(\Sigma_{a} \wedge \Sigma_{a}\right) \\
V_{0}^{4}=\frac{1}{4 !} i_{\xi} i_{\xi} i_{\xi} i_{\xi}\left(\Sigma_{a} \wedge \Sigma_{a}\right) &
\end{array}
$$


The existence of the two descents above naturally leads us to ask whether a further descent can be constructed from $\widetilde{\Sigma}_{a} \wedge \widetilde{F}_{a}$ by considering $\widetilde{D}\left(\widetilde{\Sigma}_{a} \wedge \widetilde{F}_{a}\right)$. It is straightforward to verify that from

$$
\left.(d+\delta) \widetilde{\Sigma}_{a} \wedge \widetilde{F}_{a}\right)=\widetilde{D} \widetilde{\Sigma}_{a} \wedge \widetilde{F}_{a}
$$

we arrive at

$$
\begin{aligned}
\delta\left(\Sigma_{a} \wedge F_{a}\right) & =-d\left[i_{\xi}\left(\Sigma_{a} \wedge F_{a}\right)\right]+i_{\xi} d\left(\Sigma_{a} \wedge F_{a}\right) \\
\delta\left[i_{\xi}\left(\Sigma_{a} \wedge F_{a}\right)\right] & =-d\left[\frac{1}{2 !} i_{\xi} i_{\xi}\left(\Sigma_{a} \wedge F_{a}\right)\right]+\frac{1}{2 !} i_{\xi} i_{\xi} d\left(\Sigma_{a} \wedge F_{a}\right) \\
\delta\left[\frac{1}{2 !} i_{\xi} i_{\xi}\left(\Sigma_{a} \wedge F_{a}\right)\right] & =-d\left[\frac{1}{3 !} i_{\xi} i_{\xi} i_{\xi}\left(\Sigma_{a} \wedge F_{a}\right)\right]+\frac{1}{3 !} i_{\xi} i_{\xi} i_{\xi} d\left(\Sigma_{a} \wedge F_{a}\right) \\
\delta\left[\frac{1}{3 !} i_{\xi} i_{\xi} i_{\xi}\left(\Sigma_{a} \wedge F_{a}\right)\right] & =-d\left[\frac{1}{4 !} i_{\xi} i_{\xi} i_{\xi} i_{\xi}\left(\Sigma_{a} \wedge F_{a}\right)\right]+\frac{1}{4 !} i_{\xi} i_{\xi} i_{\xi} i_{\xi} d\left(\Sigma_{a} \wedge F_{a}\right) \\
\delta\left[\frac{1}{4 !} i_{\xi} i_{\xi} i_{\xi} i_{\xi}\left(\Sigma_{a} \wedge F_{a}\right)\right] & =-\frac{1}{5 !} i_{\xi} i_{\xi} i_{\xi} i_{\xi} i_{\xi} d\left(\Sigma_{a} \wedge F_{a}\right)
\end{aligned}
$$

The non-exact terms on the R.H.S. are again contractions with the ghost $\xi$ of $d\left(\Sigma_{a} \wedge F_{a}\right)$, which also vanish in four dimensions. There is thus a third BRST cohomology descent sequence. While the two previous descents involve either $F$ or $\Sigma$ but not both together, this third set of descent equations involves both $\Sigma$ and $F$ i.e. the conjugate variables $\widetilde{\sigma}$ and $A$. The corresponding descent equations can be written as

$$
\begin{aligned}
d U_{4}^{0} & =0 \\
\delta U_{4-g}^{g} & =-d U_{3-g}^{g+1} \quad \text { for } g=1,2,3, \\
\delta U_{0}^{4} & =0
\end{aligned}
$$

with

$$
\begin{array}{ll}
U_{4}^{0}=\Sigma_{a} \wedge F_{a} & U_{3}^{1}=i_{\xi}\left(\Sigma_{a} \wedge F_{a}\right) \\
U_{2}^{2}=\frac{1}{2 !} i_{\xi} i_{\xi}\left(\Sigma_{a} \wedge F_{a}\right) & U_{1}^{3}=\frac{1}{3 !} i_{\xi} i_{\xi} i_{\xi}\left(\Sigma_{a} \wedge F_{a}\right) \\
U_{0}^{4}=\frac{1}{4 !} i_{\xi} i_{\xi} i_{\xi} i_{\xi}\left(\Sigma_{a} \wedge F_{a}\right) &
\end{array}
$$

The elements of the BRST descents can be used to construct invariants by integrating over the appropriate cycles. Observables of the theory should be expressed in terms of these invariants. To obtain the invariants, consider $\gamma_{p} \in H_{p}(M) ; p=0, \ldots, 4$. Picking an element $Y_{p}^{4-p}$ of the $W, V$, or $U$ descent, we see that

$$
\begin{aligned}
\delta \int_{\gamma_{p}} Y_{p}^{4-p} & =-\int_{\gamma_{p}} d Y_{p-1}^{5-p} \\
& =-\int_{\partial \gamma_{p}} Y_{p-1}^{5-p} \\
& =0
\end{aligned}
$$


Moreover, the BRST cohomology class of $Y_{p}^{4-p}\left(\gamma_{p}\right) \equiv \int_{\gamma_{p}} Y_{p}^{4-p}$ depends only on the homology class of $\gamma_{p}$ because the descent equations guarantee that for $\gamma_{p}, \zeta_{p}=\gamma_{p}+\partial \omega_{p+1} \in$ $H_{p}(M) ; p=0, \ldots, 3$

$$
\begin{aligned}
Y_{p}^{4-p}\left(\zeta_{p}\right) & =\int_{\gamma_{p}+\partial \omega_{p+1}} Y_{p}^{4-p} \\
& =Y_{p}^{4-p}\left(\gamma_{p}\right)+\int_{\omega_{p+1}} d Y_{p}^{4-p} \\
& =Y_{p}^{4-p}\left(\gamma_{p}\right)-\delta \int_{\omega_{p+1}} Y_{p+1}^{3-p}
\end{aligned}
$$

Finally, consider the following transformation:

$$
\delta \int_{M} S^{a b} F_{a} \wedge F_{b}=-\int_{\partial M} i_{\xi}\left(S^{a b} F_{a} \wedge F_{b}\right)
$$

Provided the boundary term vanishes (which is automatic if $M$ has no boundary), a further global invariant, $\int_{M} S^{a b} F_{a} \wedge F_{b}$, will be present. In the classical context, this is an independent invariant only for Petrov Type I Einstein manifolds[11]. It is possible to construct a descent for which $S^{a b} F_{a} \wedge F_{b}$ is the zero ghost number four-form. This can be achieved by using the identity

$$
\begin{aligned}
\widetilde{D}\left(S^{a b} \widetilde{F}_{a} \wedge \widetilde{F}_{b}\right) & =\left(\widetilde{D} S^{a b}\right) \wedge \widetilde{F}_{a} \wedge \widetilde{F}_{b} \\
& =\left[(D S)^{a b}+i_{\xi}(D S)^{a b}\right] \wedge \widetilde{F}_{a} \wedge \widetilde{F}_{b}
\end{aligned}
$$

with $S_{a b}=-\frac{1}{4} *\left(F_{a} \wedge \Sigma_{b}+\Sigma_{a} \wedge F_{b}\right)$. The descent equations take the form

$$
\begin{aligned}
& d\left(S^{a b} F_{a} \wedge F_{b}\right)=0 \\
& \delta\left(S^{a b} F_{a} \wedge F_{b}\right)=-d\left[i_{\xi}\left(S^{a b} F_{a} \wedge F_{b}\right)\right] \\
& \delta\left[i_{\xi}\left(S^{a b} F_{a} \wedge F_{b}\right]=-d\left[\frac{1}{2 !} i_{\xi} i_{\xi}\left(S^{a b} F_{a} \wedge F_{b}\right)\right]\right. \\
& \delta\left[\frac{1}{2 !} i_{\xi} i_{\xi}\left(S^{a b} F_{a} \wedge F_{b}\right)\right]=-d\left[\frac{1}{3 !} i_{\xi} i_{\xi} i_{\xi}\left(S^{a b} F_{a} \wedge F_{b}\right)\right] \\
& \delta\left[\frac{1}{3 !} i_{\xi} i_{\xi} i_{\xi}\left(S^{a b} F_{a} \wedge F_{b}\right)\right]=-d\left[\frac{1}{4 !} i_{\xi} i_{\xi} i_{\xi} i_{\xi}\left(S^{a b} F_{a} \wedge F_{b}\right)\right] \\
& \delta\left[\frac{1}{4 !} i_{\xi} i_{\xi} i_{\xi} i_{\xi}\left(S^{a b} F_{a} \wedge F_{b}\right)\right]=0
\end{aligned}
$$

Unlike the previous descents, this set of equations explicitly involves the duality operator *, and hence the inverse of the metric $g_{\mu \nu}$, defined through Eqn. (3). Since degenerate metrics could not be ruled out in quantum fluctuations, and the descent involves complicated products of non-commuting operators, it remains to be seen whether the descent 
(41 )survives regularization. However, even in the classical context, it would be interesting to investigate the interplay between degenerate metrics and the invariants defined by (41).

In TQFT, the form with zero ghost number in the descent equations, called the topform, can be regarded as the action before gauge fixing. For our purposes, the action (1) is also a combination of top-forms from two of the three sequences, and correspond to $U_{4}^{0}$, and the cosmological(volume) 4 -form $V_{4}^{0}$. The last top-form, $W_{4}^{0}$ can be added on to the action density, and will give a term analogous to the $\theta$-term in QCD.

A Dirac quantization of the theory requiring that physical quantum states be annihilated by all the constraints has to face the problem of having a consistent ordering of the quantum constraints. We choose one specific scheme of ordering to make the discussion concrete. While such a procedure is not the final word on such a complicated theory, it is nevertheless instructive, and one expects the broad features of the theory to be present for all schemes. An ordering with a formal closure of the quantum constraint algebra exists and it can be shown 12] that the resulting physical states selected by

$$
\widehat{H}\left|\Psi>=\widehat{G}^{a}\right| \Psi>=\widehat{H}_{i} \mid \Psi>=0
$$

has a sector described by

$$
\widehat{Q} \mid \Psi>=0
$$

where

$$
\widehat{Q}=\frac{\lambda}{3} \int_{M^{3}} d^{3} x \delta A_{i a} \exp \left(-i \frac{3 C}{\lambda}\right) \frac{\delta}{2 i \delta A_{i a}} \exp \left(i \frac{3 C}{\lambda}\right)
$$

and $C$ denotes the Chern-Simons functional

$$
C=\int_{M^{3}} d^{3} x \epsilon^{i j k}\left(A_{i a} \partial_{j} A_{k}^{a}+\frac{1}{3} \epsilon^{a b c} A_{i a} A_{j b} A_{c k}\right)
$$

Indeed in the reduced phase space analysis, the restricted sector of the theory corresponding to (43) is described by the Ashtekar-Renteln ansatz [14]

$$
B^{i a}=-\frac{\lambda}{3} \tilde{\sigma}^{i a}
$$

This ansatz is the set of initial data for conformally self-dual Einstein manifolds and they are described by

$$
F_{a}=-\frac{\lambda}{3} \Sigma_{a}
$$


$S_{a b}=-(\lambda / 3) \delta_{a b}$ is precisely Type $\mathrm{O}$ for this sector of the full theory. A naive counting of the number of constraints tells us that if we are restricted to this TQFT phase, there are no local degrees of freedom. In this phase, the reduced action is

$$
\mathcal{A}_{T Q F T}=-\frac{3}{16 \pi \lambda G} \int_{M} F_{a} \wedge F_{a}
$$

The resulting constraints are Eqn. (46) and Gauss' law. This set of constraints, however, is reducible since (46) generates deformations of the gauge potential, and so if it holds, so will Gauss' law. The BRST analysis of this TQFT action has been performed (see[6] for details) and the BRST invariants from the descent equations were successfully identified with the Donaldson maps by Witten [4]. If we let $\delta_{T Q F T}$ be the BRST transformation, then the action is invariant under

$$
\begin{aligned}
\delta_{T Q F T} A_{a} & =-D \eta_{a}+\psi_{a} \\
\delta_{T Q F T} \eta_{a} & =-\frac{1}{2} \epsilon_{a}{ }^{b c} \eta_{b} \eta_{c}+\phi_{a} \\
\delta_{T Q F T} \psi_{a} & =-\epsilon_{a}{ }^{b c} \eta_{b} \psi_{c}-D \phi_{a} \\
\delta_{T Q F T} \phi_{a} & =-\epsilon_{a}{ }^{b c} \eta_{b} \phi_{c}
\end{aligned}
$$

Comparing with Eqns. (20) and (21), this restricted (Type O) phase of the full theory can be identified with the TQFT of Eqn. (48) with Donaldson-Witten invariants, if we make the substitution

$$
\psi_{a}=i_{\xi} F_{a} \quad \text { and } \quad \phi_{a}=\frac{1}{2 !} i_{\xi} i_{\xi} F_{a}
$$

In this sector, the role of $\Sigma$ has been eliminated in terms of $F$. From the vantage point of the BRST invariants and the observables we have constructed so far, we see that in this phase the elements of the descents are degenerate because of (47).

Eqn. (42) can have solutions which are not annihilated by the topological charge $Q$ of Eqns. (44) - (45). When this happens, we are no longer in the TQFT phase, and classically at least, we will have solutions which are not of Type O. Outside of this phase, the variables $\Sigma$ and $F$ are no longer proportional to each other, and so there can be full-fledged canonical degrees of freedom in the theory. A naive counting of the number of constraints minus the number of conjugate pairs shows that we now have up to two unconstrained degrees of freedom. These represent local fluctuations in the vierbeins and the Ashtekar connections [11]. However, it is important to remember that we still maintain complete diffeomorphism invariance, so it is not clear these degrees of freedom can be 
directly identified with the graviton [4] [5]. In any case, the BRST descent sequences are now independent of each other, and the observables are characterized by many more invariants. Despite the local nature of the fluctuations in $\Sigma$ and $F$, the observables described by the invariants are non-local, since exact diffeomorphism symmetry remains unbroken. The physical implications of these observables when this invariance is broken, as well as the important question of what happens in the presence of matter couplings, will be discussed elsewhere.

\section{Acknowledgments}

We thank Marek Grabowski and Waichi Ogura for helpful discussions. 


\section{References}

[1] A. M. Polyakov, Mod.Phys.Lett. A11, (1987)801.

[2] E. Witten, Nucl.Phys. B311, (1988)46.

[3] For a review, see S. K. Donaldson and P. B. Kronheimer, The geometry of fourmanifolds, (Oxford Science Publications, Clarendon Press, Oxford, 1990).

[4] E. Witten, Comm. Math. Phys. 117, (1988)353.

[5] E. Witten, Comm.Math.Phys. 118, (1988)411.

[6] This subject is reviewed in D. Birmingham, M. Blau, M. Rakowski, and G. Thompson, Phys. Rep. 209, (1991)129.

[7] S. K. Donaldson, Topology 29, (1990)257.

[8] A. Ashtekar, Phys. Rev. Letts. 57, 2244(1986); Phys. Rev. D36, 1587(1986); New perspectives in canonical gravity, (Bibliopolis, Naples, 1988); Lectures on non-perturbative canonical gravity, (World Scientific, Singapore, 1991).

[9] J. Samuel, Pramāna J. Phys. 28, (1987)L429; Class. Quant. Grav. 5, (1988)L123.

[10] R. Arnowitt, S. Deser, and C. W. Misner, Phys.Rev. 120, (1960)313; Ann.Phys. 33, (1965)33.

[11] L. N. Chang and C. P. Soo, VPI-IHEP 92-5, Classical and quantum gravity in Ashtekar variables.

[12] H. Kodama, Phys.Rev. D42, (1990)2548.

[13] L. N. Chang and C. P. Soo, VPI-IHEP 91-2, Ashtekar's variables and the topological phase of quantum gravity, Proceedings of the XXth. Conference on Differential Geometric Methods in Physics, ed. S. Catto, A. Rocha, (World Scientific, Singapore, 1991.)

[14] A. Ashtekar and P. Renteln, Lecture notes on new variables, Astrophys. Grp., Math.Dept., University of Poona (1987). 